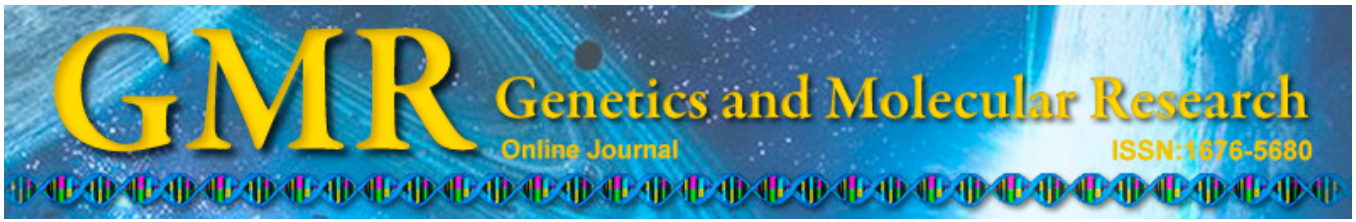

\title{
Association between the ERCC2 rs13181 polymorphism and the risk of glioma: a meta-analysis
}

\author{
T.L. Jia ${ }^{1}$, H.J. Wu ${ }^{1}$, H.B. Wang ${ }^{1}$, W.B. $\mathrm{Ma}^{2}$ and B. Xing ${ }^{2}$ \\ ${ }^{1}$ Department of Neurosurgery, the First Central Hospital of Baoding, \\ Baoding, China \\ ${ }^{2}$ Department of Neurosurgery, Peking Union Medical College Hospital, \\ Peking Union Medical College, Chinese Academy of Medical Sciences, \\ Beijing, China \\ Corresponding author: B. Xing \\ E-mail: xingbing_pumc@163.com \\ Genet. Mol. Res. 14 (4): 12577-12584 (2015) \\ Received May 14, 2015 \\ Accepted August 25, 2015 \\ Published October 19, 2015 \\ DOI http://dx.doi.org/10.4238/2015.October.19.1
}

\begin{abstract}
Several studies have focused on the association between the $E R C C 2$ rs 13181 polymorphism and glioma risk, but the results were inconclusive. We aimed to conduct a meta-analysis to investigate the role of ERCC2 rs 13181 on the risk of glioma. We searched and collated the relevant studies in both Chinese and English through the PubMed, Web of Science, Cochrane Library, and EMBASE databases published through June 1, 2014. A total of 11 studies for ERCC2 rs13181 were selected; these included 3456 glioma cases and 4957 controls. Using fixed-effects model analysis, we found that no significant difference could be identified between the ERCC2 rs13181 polymorphism and the risk of glioma. Subgroup analysis showed that the ERCC2 rs13181 GT and TT genotypes were significantly associated with an increased risk of glioma in the Chinese population [odds ratio $(\mathrm{OR})=1.47$, $95 \%$ confidence interval $(\mathrm{CI})=1.17-1.85 ;$ and $\mathrm{OR}=1.50,95 \% \mathrm{CI}=$ 1.02-2.22, respectively], but no significant increased risk of glioma was detected with these genotypes in the Caucasian populations. No
\end{abstract}


publication bias was identified in this meta-analysis. Our meta-analysis strongly suggested that ERCC2 rs13181 was associated with a higher susceptibility to glioma in the Chinese population.

Key words: ERCC2; rs13181; Glioma; Meta-analysis

\section{INTRODUCTION}

Gliomas account for more than $70 \%$ of all brain tumors, which are the most common primary brain tumor in adults. The prognosis of glioma is considered to be poor when compared with other types of brain tumors (Bondy et al., 2008). It is well known that many environmental and lifestyle factors play an important role in the development of glioma, such as occupational factors, ionizing radiation, cellular phone use, smoking habits, and diet, as well as genetic factors (Ohgaki and Kleihues, 2005; Goodenberger and Jenkins, 2012). However, the exact etiology of glioma is still not well understood. Previous studies reported that many genetic factors could affect the risk of glioma, including CCDC26, CCND1, CHEK2, GSTP1, P53, and ERCC1 (Dong et al., 2014; Qin et al., 2014; Wei et al., 2014; Zhu et al., 2014).

In normal cells, DNA is subjected to many kinds of assaults such as ionizing radiation, ultraviolet rays, and genotoxic agents. Therefore, the DNA repair function can have a substantial role in preventing the propagation of errors and maintaining genomic stability (Vogelstein and Kinzler, 2004). The process of DNA repair includes about 130 genes and several molecular pathways, such as the base-excision repair, nucleotide excision repair (NER), and homologous recombination repair pathways (Popanda et al., 2004). Variations in DNA repair gene sequence can alter the expression of the gene itself and the related protein, and therefore can influence the efficiency of DNA repair and increase the susceptibility to carcinogenesis. Many genes in the DNA repair pathways have important roles in the maintenance of genomic integrity and fidelity, and previous studies have investigated the relationship between polymorphisms in DNA repair genes and the risk of glioma. Among these genes, excision repair cross-complementing group 2 (ERCC2) is considered to have a role in the development of glioma. ERCC2 is reported to act as the rate-limiting enzyme in the process of NER, and is located at chromosome 19q13.3 (McKean-Cowdin et al., 2009). Several previous studies have reported the association between variants of the ERCC2 rs13181 polymorphism and the risk of glioma, but the results of these studies have been inconsistent (McKean-Cowdin et al., 2009; Gao et al., 2014; Rodriguez-Hernandez et al., 2014). Therefore, we aimed to conduct a metaanalysis to investigate the role of ERCC2 rs13181 on the risk of glioma.

\section{MATERIAL AND METHODS}

\section{Search strategy}

A comprehensive search was conducted through the databases of PubMed, EMBASE, and the China National Knowledge Infrastructure platforms until June 1, 2014. The search strategies were conducted including the following terms and their combinations: glioma, brain tumor, polymorphism, variant, ERCC2, and rs13181.

\section{Inclusion and exclusion criteria}

The inclusion criteria for studies were as follows: case-control design, evaluation of 
the association between the ERCC2 rs13181 polymorphism and glioma risk, and results of available genotype frequencies were included.

The exclusion criteria for studies were as follows: articles only consisted of an abstract, were review articles or comments, overlap with other studies, or contained no comparison or control group.

\section{Data extraction}

Two authors independently completed the electronic database search. Duplications and obviously irrelevant reports were excluded. Full texts of identified eligible studies were obtained using the established inclusion criteria. Disagreements were resolved through discussion. The following items were extracted for each eligible study: study ID, study design, participation number, case-control number, and genotype information.

\section{Statistical analysis}

All meta-analysis examination procedures were conducted by the STATA 9.0 software (version 9.0, USA). A chi-square $\left(\chi^{2}\right)$ test was performed to evaluate the Hardy-Weinberg equilibrium (HWE) status of control groups. The pooled odds ratios (ORs) and $95 \%$ confidence intervals (CIs) were calculated to determine the association between the ERCC2 rs13181 polymorphism and glioma risk. The heterogeneity between studies was estimated by $I^{2}$ and heterogeneity Q statistics tests. When $I^{2}$ values were in the range of $0-25 \%$, there was no degree of heterogeneity. When $I^{2}$ values were in the range of $25-50 \%$, there was moderate heterogeneity. When $I^{2}$ values were in the range of $75-100 \%$, there was great heterogeneity. A random-effects model or fixed-effects model was used to calculate the pooled OR $(95 \% \mathrm{CI})$ according to the degree of heterogeneity between studies. The publication bias in studies was conducted by Begg's funnel plot and the Egger test.

\section{RESULTS}

The initial search identified 46 studies for ERCC2 rs13181, of which 11 met the selection criteria (Caggana et al., 2001; Wrensch et al., 2005; Yang et al., 2005; Liu et al., 2009; McKean-Cowdin et al., 2009; Rajaraman et al., 2010; Chen et al., 2012; Luo et al., 2013; Salnikova et al., 2013; Zhang, 2013; Rodriguez-Hernandez et al., 2014). Among the excluded studies, four were dissertations, three were reviews, 21 did not investigate the ERCC2 rs 13181 polymorphism, eight did not report the association between the ERCC2 rs13181 polymorphism and risk of glioma, and two were replication studies. Eventually, a total of 11 studies of ERCC2 rs13181 were selected, which included 3456 glioma cases and 4957 controls. The genetic distributions of $E R C C 2$ rs 13181 variants in ten studies were in accordance with HWE. Only one study deviated from HWE in the control group (Luo et al., 2013). The meta-analysis for the association between ERCC2 rs13181 and the risk of glioma is shown in Table 1. There was no significant heterogeneity between studies, and thus we performed a fixed-effects model to assess the association between ERCC2 rs13181 and the risk of glioma. We found that no significant difference could be identified between the ERCC2 rs13181 polymorphism and a risk of glioma (Table 1). 


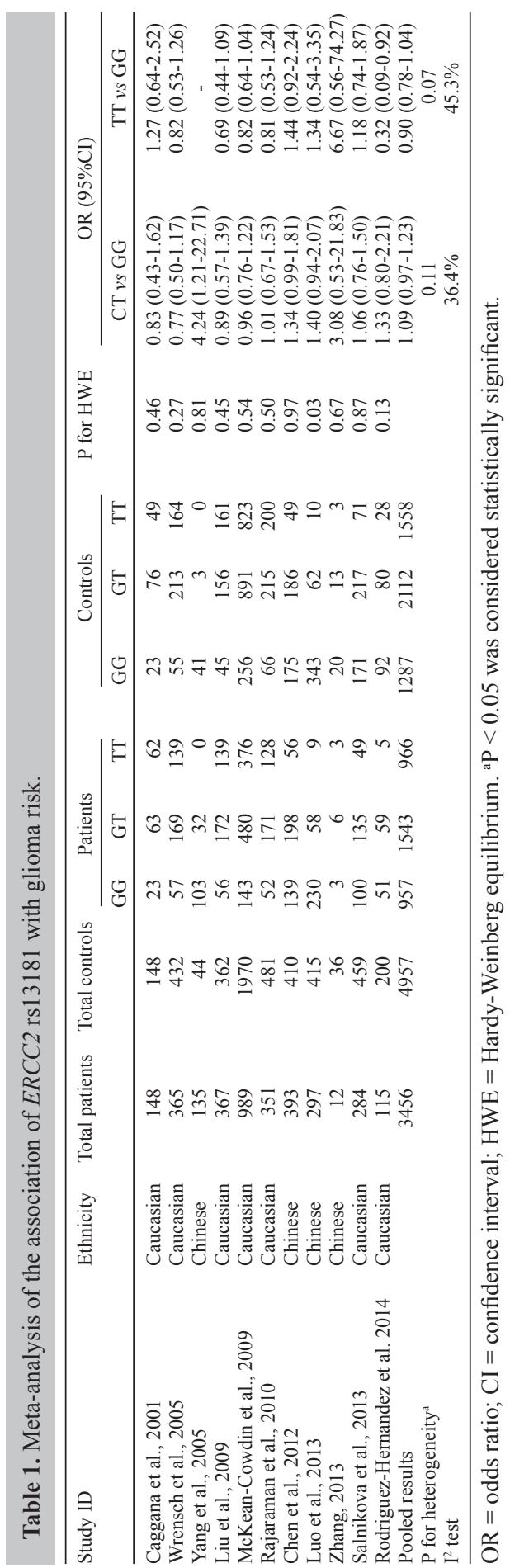


Subgroup analysis showed that ERCC2 rs13181 GT and TT genotypes were significantly associated with an increased risk of glioma in the Chinese population ( $\mathrm{OR}=1.47$, $95 \% \mathrm{CI}=1.17-1.85 ; \mathrm{OR}=1.50,95 \% \mathrm{CI}=1.02-2.22$, respectively), but no significant increased risk of glioma was identified in the Caucasian populations (Figures 1 and 2). In this study, the shapes of the funnel plots for the ERCC2 rs13181 polymorphism showed a symmetric distribution, which suggested no publication bias was present in this meta-analysis (Figure 3).

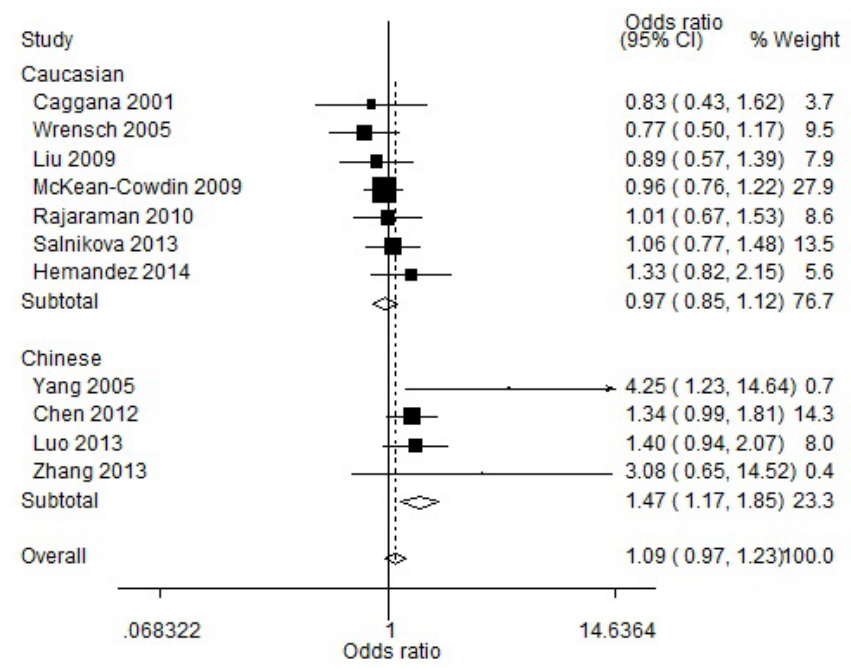

Figure 1. Subgroup analysis for the association between the ERCC1 rs13181 GT genotype and the risk of glioma. CI, confidence interval.

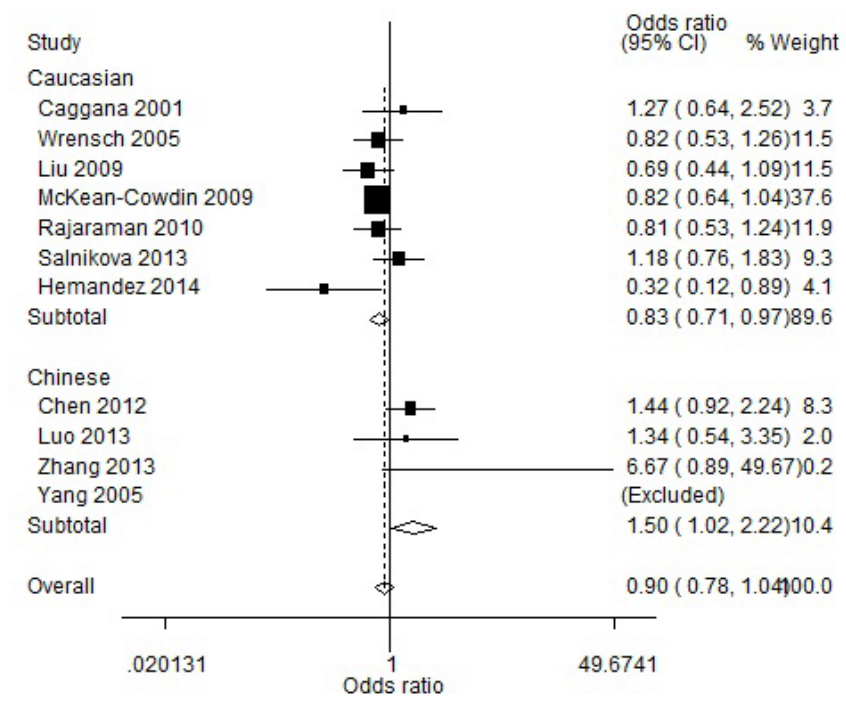

Figure 2. Subgroup analysis for the association between the ERCC1 rs13181 TT genotype and the risk of glioma. CI, confidence interval. 


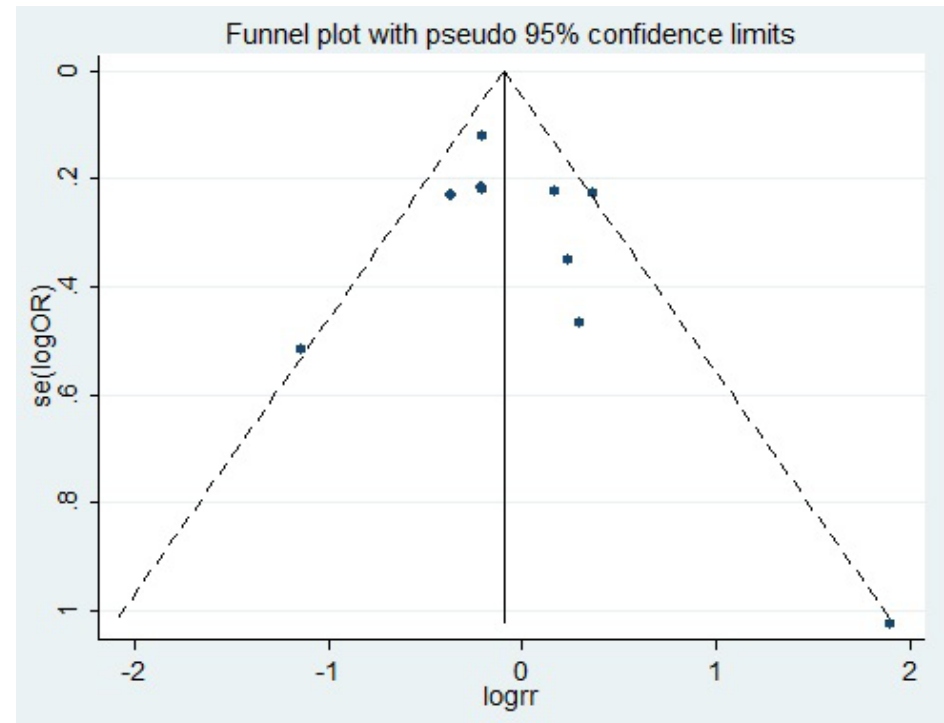

Figure 3. Funnel plot of the association between the ERCC2 rs13181 polymorphism and the risk of glioma.

\section{DISCUSSION}

The ERCC2 protein is a key element in the transcription-coupled NER pathway, and it plays an important role in altering the DNA repair capacity. ERCC2 has a key role in maintaining genetic stability, and deficits in ERCC2 proteins can influence the susceptibility to cancer (Goode et al., 2002). Previous studies have shown that the ERCC2 rs13181 polymorphism was correlated with a high level of DNA adducts or lower DNA repair capacity (Lunn et al., 2000; Qiao et al., 2002). Therefore, we investigated the association of the ERCC2 polymorphism on the risk of glioma. The results suggest that the ERCC2 rs13181 polymorphism is not associated with an overall increased risk of glioma.

The ERCC2 rs13181 polymorphic variant can cause a defect in NER, and considerable investigation has been performed on the role of insufficient DNA repair in carcinogenesis. Previous studies have demonstrated that a defect of the DNA repair function is a risk factor for several kinds of cancer, including bladder cancer, acute leukemia, and breast cancer (Yan et al., 2014; Liu et al., 2014). Li et al. (2014) reported a meta-analysis with 11 studies, including 3797 cases and 5094 controls, and showed that polymorphism in ERCC2 rs13181 contributes to bladder cancer susceptibility. Yan et al. (2014) conducted a meta-analysis with 22 studies, and reported that polymorphism in ERCC2 rs13181 significantly increased breast cancer risk. Liu et al. (2014) performed a meta-analysis with ten published case-control studies including 1494 cases and 2259 controls, and reported that the ERCC2 rs13181 variant genotype might be a risk factor for acute leukemia. Despite a growing number of epidemiologic studies that have reported an association between the ERCC2 rs13181 polymorphism and the risk of glioma, no significant association was reported between them. Caggana et al. (2001) first reported an association between the ERCC2 rs13181 polymorphism and risk of glioma in a gender- and agematched case-control study, but did not find any association between them. Another study in the United States showed that no significant association existed between the ERCC2 rs 13181 
polymorphism and the risk of adult glioma (Liu et al., 2009). However, several recent studies reported that the $E R C C 2$ rs 13181 polymorphism was associated with glioma risk (Chen et al., 2012; Zhang, 2013; Rodriguez-Hernandez, 2014). The inconsistency of these studies might be explained by differences in population background, sample size, and also by chance. Our meta-analysis showed that the ERCC2 rs13181 polymorphism increased the risk of glioma.

Although publication bias was not identified in this meta-analysis, this study has some potential limitations. First, only ten eligible studies were included in this meta-analysis. Therefore, in the subgroup analyses by ethnicity, the number of cases and controls was relatively small, which might have resulted in low statistical power to identify the association. Furthermore, different pathological types of glioma were not considered, which may be a source of betweenstudy heterogeneity. Unfortunately, only two studies in Caucasian populations provided genetic data of ERCC2 rs13181 polymorphic variation for glioblastoma multiforme and nonglioblastoma multiforme. Finally, our meta-analysis was largely performed with unadjusted estimates, because of the limitations in the studies selected that presented adjusted estimates. Although adjusted estimates were shown, the estimates were not adjusted by the same confounders. It was also difficult to present the combined estimates by adjusted potential confounders.

In conclusion, our meta-analysis strongly suggested that ERCC2 rs13181 was associated with a higher susceptibility to glioma in the Chinese population. Studies with larger sample size and more specified information on pathological types of glioma are needed to confirm our results for both Chinese and Caucasian populations.

\section{REFERENCES}

Bondy ML, Scheurer ME, Malmer B, Barnholtz-Sloan JS, et al. (2008). Brain tumor epidemiology: consensus from the Brain Tumor Epidemiology Consortium. Cancer 113: 1953-1968.

Caggana M, Kilgallen J, Conroy JM, Wiencke JK, et al. (2001). Associations between ERCC2 polymorphisms and gliomas. Canc. Epidemiol. Biomarkers Prev. 10: 355-360.

Chen DQ, Yao DX, Zhao HY and Yang SJ (2012). DNA repair gene ERCC1 and XPD polymorphisms predict glioma susceptibility and prognosis. Asian Pac. J. Canc. Prev. 13: 2791-2794.

Dong YS, Hou WG, Li XL, Jin TB, et al. (2014). Genetic association of CHEK2, GSTP1, and ERCC1 with glioblastoma in the Han Chinese population. Tumour Biol. 35: 4937-4941.

Gao K, Mu SQ and Wu ZX (2014). Investigation of the effects of single-nucleotide polymorphisms in DNA repair genes on the risk of glioma. Genet. Mol. Res. 13: 1203-1211.

Goode EL, Ulrich CM and Potter JD (2002). Polymorphisms in DNA repair genes and associations with cancer risk. Cancer Epidemiol. Biomarkers Prev. 11: 1513-1530.

Goodenberger ML and Jenkins RB (2012). Genetics of adult glioma. Canc. Genet. 205: 613-621.

Li SX, Dai QS, Chen SX, Zhang SD, et al (2014). Xeroderma pigmentosum complementation group D (XPD) gene polymorphisms contribute to bladder cancer risk: a meta-analysis. Tumour Biol. 35: 3905-3915.

Liu D, Wu D, Li H and Dong M (2014). The effect of XPD/ERCC2 Lys751Gln polymorphism on acute leukemia risk: A systematic review and meta-analysis. Gene 538: 209-216.

Liu Y, Scheurer ME, El-Zein R, Cao Y, et al. (2009). Association and interactions between DNA repair gene polymorphisms and adult glioma. Canc. Epidemiol. Biomarkers Prev. 18: 204-214.

Lunn RM, Helzlsouer KJ, Parshad R, Umbach DM, et al. (2000). XPD polymorphisms: effects on DNA repair proficiency. Carcinogenesis 21: 551-555.

Luo KQ, Mu SQ, Wu ZX, Shi YN, et al. (2013). Polymorphisms in DNA repair genes and risk of glioma and meningioma. Asian Pac. J. Canc. Prev. 14: 449-452.

McKean-Cowdin R, Barnholtz-Sloan J, Inskip PD, Ruder AM, et al. (2009). Associations between polymorphisms in DNA repair genes and glioblastoma. Canc. Epidemiol. Biomarkers Prev. 18: 1118-1126.

Ohgaki H and Kleihues P (2005). Epidemiology and etiology of gliomas. Acta Neuropathol. 109: 93-108.

Popanda O, Schattenberg T, Phong CT, Butkiewicz D, et al. (2004). Specific combinations of DNA repair gene variants and increased risk for non-small cell lung cancer. Carcinogenesis 25: 2433-2441.

Qiao Y, Spitz MR, Shen H, Guo Z, et al. (2002). Modulation of repair of ultraviolet damage in the host-cell reactivation 
assay by polymorphic XPC and XPD/ERCC2 genotypes. Carcinogenesis 23: 295-299.

Qin LY, Zhao LG, Chen X, Li P, et al. (2014). The CCND1 G870A gene polymorphism and brain tumor risk: a metaanalysis. Asian Pac. J. Cancer Prev. 15: 3607-3612.

Rajaraman P, Hutchinson A, Wichner S, Black PM, et al. (2010). DNA repair gene polymorphisms and risk of adult meningioma, glioma, and acoustic neuroma. Neuro. Oncol. 12: 37-48.

Rodriguez-Hernandez I, Perdomo S, Santos-Briz A, Garcia JL, et al. (2014). Analysis of DNA repair gene polymorphisms in glioblastoma. Gene 536: 79-83.

Salnikova LE, Belopolskaya OB, Zelinskaya NI and Rubanovich AV (2013). The potential effect of gender in CYP1A1 and GSTM1 genotype-specific associations with pediatric brain tumor. Tumour Biol. 34: 2709-2719.

Vogelstein B and Kinzler KW (2004). Cancer genes and the pathways they control. Nat. Med. 10: 789-799.

Wei XB, Jin TB, Li G, Geng TT, et al. (2014). CCDC26 gene polymorphism and glioblastoma risk in the Han Chinese population. Asian Pac. J. Cancer

Prev. 15: 3629-3633.

Wrensch M, Kelsey KT, Liu M, Miike R, et al. (2005). ERCC1 and ERCC2 polymorphisms and adult glioma. Neuro. Oncol. 7: 495-507.

Yan Y, Liang H, Light M, Li T, et al. (2014). XPD Asp312Asn and Lys751Gln polymorphisms and breast cancer susceptibility: a meta-analysis. Tumour Biol. 35: 1907-1915.

Yang D, Li QG, Zhang YZ, Wang HY, et al. (2005). Comparison of screen for single nucleotide polymorphisms of the glioma susceptibility gene - ERCC2 by using denaturing high performance liquid chromatography and restriction fragment length polymorphism. Chin. J. Min. Invas. Neurosurg. 10: 164-166.

Zhang X (2013). Correlation of ERCC2 K751Q polymorphism, EGF +61G/A polymorphism and the risk of glioma. Soochow University.

Zhu W, Lu L, Li Y, Yao J, et al. (2014). The effects of p53 Arg72Pro polymorphism on glioma susceptibility: a metaanalysis. Tumour Biol. 35: 3725-3730. 